Ger J Exerc Sport Res 2020 · 50:511-522 https://doi.org/10.1007/s12662-020-00675-6 Eingegangen: 2. März 2020

Angenommen: 14. August 2020

Online publiziert: 18 . September 2020

(c) Der/die Autor(en) 2020

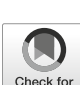

Check for
updatates

\author{
Matthias Baumgartner ${ }^{1}$ D $\cdot$ Verena Oesterhelt ${ }^{2} \cdot$ Sabine Reuker $^{3}$ \\ ${ }^{\prime}$ Institut Professionsforschung und Kompetenzentwicklung, Pädagogische Hochschule St.Gallen, \\ St.Gallen, Schweiz \\ ${ }^{2}$ Institut für Sportwissenschaft, Professur für Sportwissenschaft mit Schwerpunkt Sportdidaktik, Justus- \\ Liebig-Universität Giessen, Giessen, Deutschland \\ ${ }^{3}$ Institut für Kunst, Musik und Sport, Abteilung Sport - Sportpädagogik, Pädagogische Hochschule \\ Ludwigsburg, Ludwigsburg, Deutschland
}

\section{Konstruktion und Validierung eines multidimensionalen Beobachtungsinstruments zur Erfassung der klassenführungs- bezogenen Performanzen von sportunterrichtenden Lehrkräften (KlaPe-Sport)}

\section{Einleitung}

Das Klassenmanagement bzw. die Klassenführung von (sportunterrichtenden) Lehrkräften gilt unbestritten als ein bedeutsamer Faktor eines guten (Sport-)Unterrichts (Gettinger \& Kohler, 2006; Heemsoth, 2014; Piwowar, 2013). In Anlehnung an ein integratives Kompetenzverständnis (Baumgartner, 2018a) ist davon auszugehen, dass die hier interessierende beobachtbare klassenführungsbezogene Performanz (klassenführungsbezogene Könnensleistungen) eine bedeutsame Kompetenzfacette des Kompetenzbereichs der Klassenführung darstellt. ${ }^{1}$ Aus der vorliegenden Forschungsliteratur wird ersichtlich, dass die Klassenführung sehr unterschiedlich bestimmt und erfasst wird

\footnotetext{
1 Im Sinn eines integrativen Verständnisses beinhaltet ein Kompetenzbereich die drei Kompetenzfacetten a) Aspekte professioneller Kompetenz (z. B. professionelles Wissen; Baumert, \& Kunter, 2006), situierte Wahrnehmungs-, Interpretations- und Entscheidungsfähigkeiten (Reuker, 2017a, 2017b, 2018) sowie c) beobachtbare Performanz, die aus den beiden anderen Facetten hervorgeht (Baumgartner, 2018a).
}

(z.B. Helmke, 2017). Mit der Zielsetzung, ein Beobachtungsinstrument für klassenführungsbezogene Performanzen von sportunterrichtenden Lehrkräften $\mathrm{zu}$ entwickeln, begrenzen wir uns im Folgenden auf solche Merkmale, die im Sportunterricht zu beobachten sind. Handlungen von sportunterrichtenden Lehrkräften, die in der Situation nicht direkt beobachtbar sind, da sie zu einem früheren Zeitpunkt eingeführt wurden oder aus dem Unterricht ausgelagert werden (z. B. Erstellung eines langfristigen Regelsystems; Helmke, 2017) bleiben im Folgenden unberücksichtigt. In Bezug auf diese engere Interpretation wird im folgenden Beitrag der Begriff der Klassenführung verwendet, der sich im Vergleich zum Ausdruck Klassenmanagement auf das im Sportunterricht beobachtbare klassenführungsbezogene Handeln beschränkt.

Obschon die Klassenführung von sportunterrichtenden Lehrkräften als ein relevanter Kompetenzbereich zu deuten ist (Baumgartner, 2013a; Baumgartner, Owassapian, \& Perret Guldimann, angenommen; Heemsoth, 2014; Herrmann, Seiler, \& Niederkofler, 2016), liegen keine empirisch validierten Beobachtungsinstrumente vor, mithilfe derer die Qualität der klassenführungsbezogenen Performanzen bei (angehenden) sportunterrichtenden Lehrkräften valide, reliabel und objektiv erfasst werden kann. Die Entwicklung derartiger Beobachtungsinstrumente erscheint als bedeutsam, weil diese u.a. die Grundlage zur Eruierung der Effektivität von hochschuldidaktischen Lehr-Lernarrangements darstellen (Baumgartner, 2017b). Der Einsatz von fachübergreifenden Beobachtungsinstrumenten ist nur bedingt möglich, weil die Klassenführung im Sportunterricht fachspezifische Eigenheiten aufweist (Cothran \& Kulinna, 2015), die aus Validitätszwecken nicht unberücksichtigt bleiben sollten (vgl. Abschnitt „Zur Klassenführung im (Sport-)Unterricht“). Der vorliegende Beitrag setzt hier an und zielt auf die Generierung eines Beobachtungsinstruments zur Erfassung der Qualität der klassenführungsbezogenen Performanzen bei (angehenden) sportunterrichtenden Lehrkräften (KlaPeSport) verschiedener Schulstufen. Der Fokus wird auf die Erhebung der klas- 
senführungsbezogenen Performanzen und beispielsweise nicht auf die Messung der Kompetenzfacette des klassenführungsbezogenen Wissens - gelegt, um eine ökologisch valide, verhaltensnahe und situierte Kompetenz- bzw. Performanzdiagnostik zu ermöglichen (Baumgartner, 2017a, 2018b).

\section{Zur Klassenführung im (Sport-)Unterricht}

Klassenführung von (sportunterrichtenden) Lehrkräften umfasst die Gesamtheit unterrichtlicher Maßnahmen, die im Kern auf die Schaffung lernförderlichen Umgebungen abzielen, um sicherzustellen, dass kognitives, sozialemotionales und motorisches Lernen möglich ist (Kulinna, Silverman, \& Keating, 2000). Klassenführung verfolgt dabei keinen Selbstzweck, sondern ist als Mittel zur Förderung der Entwicklung der Schülerinnen und Schüler zu betrachten (Baumgartner, 2017b). Im Vergleich zu anderen Unterrichtsfächern weist der Sportunterricht jedoch fachspezifische Eigenheiten auf (z.B. Cothran \& Kulinna, 2015; Oesterhelt, Gerlach, Grimminger-Seidensticker, \& Friedrich, 2020): Aufgrund der geringen räumlichen Vorstrukturierung in der Sporthalle oder z. B. auf dem Sportplatz, bilden Organisations- und Ablaufformen in ihren Ausprägungen der Sozial-, Raum-, Zeit- und Materialordnung (Funke-Wieneke, 2007) eine zentrale Anforderung bei Herstellung und Erhalt des Unterrichtsrahmens. Des Weiteren ist die Qualität der Akustik häufig gering (Cothran \& Kulinna, 2015; Wegener, Wegener, \& Kastrup, 2012), es wird eine große Menge an Materialien (Groß- und Kleingeräte) benötigt und aufgrund der exponierten Stellung des Körpers spielen die physische und psychische Verletzbarkeit der Schülerinnen und Schüler (Miethling \& Krieger, 2004) eine besondere Rolle, wodurch spezifische Sicherheits- bzw. organisatorische Maßnahmen ergriffen werden müssen. Diese Besonderheiten heben die Bedeutung der Klassenführung gerade für sportunterrichtenden Lehrkräften hervor, was auch in verschiedenen Studien bestätigt wird (Baumgartner, 2013b; Hill
\& Brodin, 2004). Dementsprechend lässt sich vermuten, dass die Klassenführung im Sportunterricht im Vergleich zu anderen Fächern nicht nur andersartig, sondern auch komplexer ist (ChepyatorThomson \& Liu, 2003).

Mit dieser Einordnung der Klassenführung in den Kontext von (Sport-)Unterricht bleibt allerdings zunächst offen, welches Unterrichtsverständnis dem klassenführungsbezogenen Handeln zugrunde liegt. Im Zuge der Diskussion um ein sich wandelndes Lehr-Lernverständnis im Nachklang der großen Schulleistungsstudien scheint sich ein Konsens hinsichtlich bedeutsamer Unterrichtsmerkmale abzubilden (Reusser, 2011). Der Fokus richtet sich dabei vor dem Hintergrund eines sozialkonstruktivistischen Verständnisses von Unterricht und Lernen - im Sinne eines interaktiven, problemorientierten Prozesses gegenüber einem reproduktiven, rezeptiven Geschehen - verstärkt auf die Lernenden, die zu erwerbenden Kompetenzen sowie auf die kompetenzbezogenen Lernergebnisse (Baumberger, 2018; Reusser, 2011). Als bedeutsam werden u.a. eine individualisierte Lernbegleitung und das Vernetzen von Wissen und Können zur Gewährleistung (für das Fach Sport: erfahrungsgebundener) verständnisorientierter fachlicher Lernprozesse, die Berücksichtigung von Selbstorganisations- und Selbststeuerungsprozesse sowie die Reflexion lernzielbezogener Lernprozesse genannt (Schierz, 2013; Serwe-Pandrick, 2013). Diese Entwicklungen bringen auch neue Anforderungen an die Klassenführung mit sich. Weinstein (1999) spricht hier von einem neuen Paradigma hin zu einem Klassenmanagement, das die Bedürfnisse von Lernenden stärker mit einbezieht und dabei u. a. auch selbstständiges Lernen ermöglicht. Eine solche Öffnung von Unterricht ist dabei nicht per se mit einem weniger systematischen Vorgehen verknüpft. So verweisen Bohl und Kucharz (2010) darauf, dass die aktive Lernzeit auch im offenen Unterricht nicht automatisch einsetzt, sondern entsprechender Klassenführung bedarf und heben damit deren Bedeutung hervor. Unter Bezugnahme auf die Merkmale von Kounin (2006) zeigen sie da- bei sowohl Gemeinsamkeiten als auch Unterschiede zur Klassenführung im klassischen lehrerzentrierten Unterricht auf. Systematische Überlegungen richten sich dabei deutlich stärker auf die Gestaltung der Lernorganisation und weniger der Instruktion. Diese etwas andere Charakteristik der Klassenführung im offenen Unterricht ist bislang allerdings noch wenig erforscht. Inwieweit die bis dato identifizierten Merkmale einer guten Klassenführung auch unter Berücksichtigung von Kontextbedingungen weiter $\mathrm{zu}$ spezifizieren und $\mathrm{zu}$ ergänzen sind, wird zukünftig noch zu klären sein (Haag, 2018). Dies gilt in ähnlicher Weise auch für Fragen der Notwendigkeit einer schulformspezifischen Klassenführung. Allerdings kann Weinert und Helmke (1997) zufolge gelingender Unterricht auf sehr unterschiedliche (situierte) Weise realisiert werden - wenngleich nicht auf beliebige. Ausgehen lässt sich demnach von grundlegenden Dimensionen, die trotz unterschiedlicher Unterrichtszugänge für die Unterrichtsqualität bedeutsam sind.

Hinsichtlich den weitreichenden Untersuchungen zur Thematik der Klassenführung geht hervor, dass eine erfolgreiche Klassenführung von Lehrkräften die Schulleistungen mit einem mittleren bis starken Effekt positiv beeinflusst (Hattie, 2009). Eine hohe Qualität der Klassenführung steht zudem in positiver Korrelation zur Aufmerksamkeit, zum Engagement und zur Motivation der Schülerinnen und Schüler (Oliver, Wehby, \& Reschly, 2011), bewirkt weniger Unterrichtsstörungen (Kounin, 2006), fördert die sozioemotionale Kompetenz der Schülerinnen und Schüler und führt in der Berufseinstiegsphase zu einem geringeren Belastungsempfinden der Lehrkräfte (Dicke, Ellig, Schmeck, \& Leutner, 2015). Schwierigkeiten in der Realisierung einer guten Klassenführung stehen in positiver Korrelation zur Arbeitsunzufriedenheit und zum Burnout-Syndrom von Lehrkräften (Brouwers \& Tomic, 2000). Entsprechende Erkenntnisse liegen auch für sportunterrichtende Lehrkräfte vor, in denen ein positiver Zusammenhang zwischen Klassenführung und der Qua- 
Ger J Exerc Sport Res 2020 · 50:511-522 https://doi.org/10.1007/s12662-020-00675-6

(c) Der/die Autor(en) 2020

\section{Baumgartner $\cdot$ V. Oesterhelt $\cdot$ S. Reuker}

\section{Konstruktion und Validierung eines multidimensionalen Beobachtungsinstruments zur Erfassung der klassenführungsbezogenen Performanzen von sportunterrichtenden Lehrkräften (KlaPe-Sport)}

\section{Zusammenfassung}

Obschon die Klassenführung von sportunterrichtenden Lehrkräften ein bedeutsamer Faktor eines guten Sportunterrichts darstellt, liegen keine Beobachtungsinstrumente vor, um die Qualität der klassenführungsbezogenen Performanzen bei sportunterrichtenden Lehrkräften valide, reliabel und objektiv erheben zu können. Derartige Instrumente erscheinen jedoch als wünschenswert, weil damit die Qualität der Klassenführung von sportunterrichtenden Lehrkräften ökologisch valide, situiert und verhaltensnah erfasst werden kann. In vorliegender Studie wurde dementsprechend die Fragestellung bearbeitet, durch welche Qualitätskriterien sich die klassenführungsbezogenen Performanzen bei sportunterrichtenden Lehrkräften erfassen lassen. In einem ersten Schritt wurde dazu eine Literaturanalyse realisiert und ein provisorisches Beobachtungsinstrument entwickelt. In einem zweiten Schritt wurde das Instrument im Rahmen einer DelphiRunde und im Sinne einer inhaltlichen Validierung Sportdidaktikerinnen und Sportdidaktikern $(n=8)$ vorgelegt. Latente und manifeste Variablen wurden dabei verändert, ausgeschlossen und neue Variablen generiert. Zur empirischen Validierung wurde in einem dritten Schritt ein Beurteilungsverfahren durchgeführt. Raterinnen und Rater $(n=6)$ absolvierten dazu ein Training. Im Anschluss wurde die Qualität der klassenführungsbezogenen Performanzen anhand von 166 Unterrichtsstunden bei angehenden und tätigen sportunterrichtenden Lehrkräften beurteilt. Zur Überprüfung der Güte des
Instruments wurden Faktorenanalysen durchgeführt und die Interraterreliabilität berechnet. Aus der empirischen Überprüfung geht ein 9-faktorielles Modell hervor, welches aus 27 manifesten Variablen besteht. Die empirische Lösung weist auf einen ausreichenden bis guten Modell-Fit, die Interraterreliabilität zeigt gute bis exzellente Kennwerte. Mit der Studie wurde ein multidimensionales Beobachtungsinstrument entwickelt, mit dem die klassenführungsbezogenen Performanzen von sportunterrichtenden Lehrkräften valide, reliabel und objektiv erfasst werden können.

Schlüsselwörter

Sportunterricht · Delphi-Studie $\cdot$ Gemischte Methode $\cdot$ Kompetenz $\cdot$ Ausbildung von Lehrkräften

\section{Development and validation of a multidimensional observation instrument for recording classroom management-related performances of physical education teachers (KlaPe-Sport)}

\section{Abstract}

Although advanced classroom management skills of physical education teachers (PET) are considered to be crucial to ensure good physical education, there are no observation tools available to assess the quality of PET classroom management-related performances in a valid, reliable and objective manner; however, such observation tools appear desirable because they enable the quality of PET classroom management skills to be recorded in an ecologically valid and context-appropriate way. Thus, this study sought to identify the quality criteria which enable an appropriate assessment of PET classroom managementrelated performance. In a first step, a review of the literature and available observation tools was carried out and a provisional observation tool was developed. In a second step, a Delphi round was conducted with physical education experts $(n=8)$ to achieve content validation of the tool. Thereby, latent variables and items were changed, excluded and new items were generated. In a third step, an evaluation procedure was carried out for empirical validation. Raters $(n=6)$ completed a rater training for this purpose. Subsequently, the quality of PET classroom management-related performances was assessed on the basis of 166 teaching hours by apprentice and senior PETs. To verify the scientific quality criteria of the observation tool, factor analyses were carried out and interrater reliability was calculated. Based on the results of this empirical evaluation, a model with 9 latent variables was developed to which 27 items can be subsumed. The results of this study contribute to ensuring a valid, reliable and objective assessment of classroom management skills of PETs.

Keywords Physical education - Delphi-study · Mixed methods - Competence $\cdot$ Teacher education lität der LehrerInnen-SchülerInnensowie der SchülerInnen-SchülerInnenBeziehung nachgewiesen werden konnte (Heemsoth, 2014). Des Weiteren wurde festgestellt, dass sportunterrichtende Lehrkräfte die Klassenführung zwar als relevant, jedoch auch als herausfordernd und belastend bewerten (Baumgartner, 2013a). Die Klassenführung wird in der Ausbildung von sportunterrichtenden Lehrkräften offensichtlich nur wenig berücksichtigt, worauf auch die Stu- dienergebnisse von Lavay, Henderson, French und Guthrie (2012) verweisen. Aus ihrer Untersuchung geht hervor, dass die Dozierenden von 134 untersuchten Ausbildungsstätten der Meinung sind, dass die Klassenführung ein relevanter Faktor des Sportunterrichts ist. Aus der Studie wird jedoch erkennbar, dass diesbezüglich nur sechs Institutionen Kurse im Bereich der Klassenführung anbieten. Der geringe Stellenwert der Klassenführung in der Ausbildung von sportunterrichtenden Lehrkräften wird auch in anderen Studien bestätigt (Garrahy, Cothran, \& Kulinna, 2005; Hill \& Brodin, 2004).

\section{Qualitätsdimensionen einer guten Klassenführung im (Sport-)Unterricht}

Das Konstrukt der Klassenführung wird in verschiedenen Forschungsbeiträgen durch unterschiedliche latenten Va- 
riablen erhoben (z.B. Kounin, 2006; Piwowar, 2013). Latente Variablen sind als Qualitätsdimensionen zu deuten, die durch manifeste Variablen (Items) erfasst werden. Im Folgenden werden latente Variablen dargestellt, welche aus der fachübergreifenden oder fachspezifischen Forschung extrahiert, an das Fach Sport angepasst oder neu generiert wurden. Sie bilden grundlegend ab, was wir unter der Begrifflichkeit der Klassenführung verstehen. Die im Folgenden dargestellten latenten Variablen basieren vor dem Hintergrund eines sozialkonstruktivistischen Lehr-Lernverständnis (vgl. Abschnitt „Zur Klassenführung im (Sport-)Unterricht") auf dem derzeitigen Stand zentraler, empirischer Erkenntnisse der Klassenführung, die sich in vorliegenden fachübergreifenden wie fachspezifischen Forschungsarbeiten als bedeutsam für einen lernwirksamen (Sport-)Unterricht erwiesen haben.

Die latente Variable Klarheit von Ansagen beinhaltet, wie deutlich und verständlich die Lehrkräfte die unterrichtlichen Zielsetzungen sowie Aufgaben, Übungen und weitere Unterrichtsschritte in Form von Ansagen, Fragen, Anregungen kommunizieren (Piwowar, 2013). Studien bestätigen, dass diese Variable in positiver Korrelation zu den Schulleistungen, der Zufriedenheit sowie zum Engagement der Schülerinnen und Schülern steht (Fendick, 1990; Hines, Cruickshank, \& Kennedy, 1985). Mit der latenten Variablen Gruppenmobilisierung wird zum Ausdruck gebracht, dass eine Lehrkraft immer eine Gruppe vor sich hat und dafür Sorge tragen muss, dass sich möglichst viele Schülerinnen und Schüler gleichzeitig mit Lernaufgaben auseinandersetzen. Dabei geht es darum, die Klasse als Ganzes in den Unterricht mit einzubeziehen und allen Schülerinnen und Schülern Verantwortung für den Lernprozess zu übertragen, damit sie sich möglichst lange und intensiv mit den Inhalten befassen (Kounin, 2006). Dies gilt auch für diejenigen, die nicht motorisch aktiv mit der Aufgabe beschäftigt sind. Gerade im Sportunterricht ist diese Variable zentral, da in Bewegungsphasen aufgrund der räumlichen und materiellen Bedingungen nicht immer alle Schülerinnen und Schüler gleichzei- tig motorisch aktiv sein können. Zudem wird im Sportunterricht häufig selbstständig in Kleingruppen an verschiedenen Stationen gearbeitet, an denen die Lehrkraft nicht überall präsent sein kann, sodass die Verantwortung für den Lernprozess auf die Gruppe übertragen werden muss. Mit der latenten Variable reibungslose Übergänge geht es um eine knappe und eindeutige Überleitung zwischen verschiedenen Unterrichtsphasen. Studien zeigen, dass der Gestaltung von reibungslosen Übergängen ein relevanter Stellenwert zukommt, weil dadurch die aktive Lernzeit erhöht und unterrichtsfernes Verhalten der Schülerinnen und Schüler vermieden werden können (Kounin, 2006). Insbesondere im Sportunterricht mit seinen besonderen Organisationsformen, wo zudem eine große Menge an Materialien benötigt wird, sind reibungslose Übergänge ein relevanter Faktor der Klassenführung (Cothran \& Kulinna, 2015). Als fachspezifische latenten Variable gilt der Aspekt Sicherheit, der im Sportunterricht im Vergleich zu anderen Fächern eine hohe Bedeutung zukommt (Wolters \& Kemna, 2011). Dies bestätigen auch tätige Lehrkräfte, die diesem Aspekt im Sportunterricht eine vorrangige Stellung zuschreiben (Baumgartner, 2013a). Mit dieser Variable werden alle Maßnahmen eingeordnet, mit denen die sportunterrichtenden Lehrkräfte die physische Sicherheit der am Unterricht teilnehmenden Personen gewährleisten. Eine weitere fachspezifische latente Variable, die Performanztransparenz, greift die Exposition der Lernenden durch die körperliche Aktivität in der Gruppe auf. So wird im Sportunterricht in der Regel direkt sichtbar, was Lernende können oder auch nicht können (Baumgartner, 2013a; Miethling \& Krieger, 2004). Im Sinne der psychischen Sicherheit (ebd.) und im Sinne des moralischen Verpflichtungsaspekts der Fürsorglichkeit (Oser, 1998) sollten sportunterrichtende Lehrkräfte u.a. organisatorische Maßnahmen ergreifen, damit sich die Lernenden im Sportunterricht nicht fortwährend ausgestellt fühlen. Dabei geht es um alle Maßnahmen, die helfen, eine zur Schau gestellte Körperlichkeit (z.B. Bewegungsdemonstrationen) auf ein vertretbares $\mathrm{Ma}$ zu reduzieren, um schamauslösende oder demütigende Situationen zu vermeiden (Klinge, 2009; Wiesche, 2013). Ein weiteres Spezifikum des Sportunterrichts ist der größere Bedarf an Großgeräten, Kleingeräten (z. B. Bälle) sowie weiterer Unterrichtsmaterialien (z.B. Videos, Flipchart etc.). Die latente Variable Materialnutzung umfasst entsprechend alle Maßnahmen zur sachgerechten und sicherheitsgemäßen Bereitstellung der Großgeräte, Kleingeräte und Unterrichtsmaterialien sowie deren dem Unterrichtsverlauf und dessen Zielsetzung angemessenen Einsatzes (Cothran \& Kulinna, 2015). Um sicherzustellen, dass Ansagen auch verstanden werden, ist Aufmerksamkeit seitens der Schülerinnen und Schüler bedeutungsvoll. Dies erfordert ebenfalls eine entsprechende Klassenführung, die mit der latenten Variable Umgang mit Störungen in Verbindung gebracht wird (Heemsoth, 2014). Dabei lassen sich unter Störungen all die Ereignisse einordnen, die verhindern, dass die zur Verfügung stehende Lernzeit optimal genutzt werden kann (Bohl \& Kucharz, 2010). Unterrichtsstörungen sind dabei immer ein „Ergebnis subjektiv empfundener und bewerteter Momentaufnahmen" (Pfitzner \& Schoppek, 2000 , S. 350) und sind in einen Interaktionsprozess eingebettet, der durch intrapersonale und soziokulturelle Bedingungen geprägt wird. Dies verweist auf eine Vielzahl an Umgangsmöglichkeiten, die situativ sehr unterschiedlich sein können. Studien zeigen auf, dass ein frühzeitiges Eingreifen und beiläufiges Intervenieren geeignete Strategien sein können, Unterrichtsstörungen zu vermeiden. Dabei ist auch darauf zu achten, Schülerinnen und Schüler nicht bloßzustellen (Emmer, Evertson, \& Worsham, 2003). Die latente Variable Momentum verweist auf einen sich kontinuierlich weiterentwickelnden, an die Bedürfnisse angepassten Unterrichtsverlauf, bei dem es auch innerhalb der Unterrichtsphasen $\mathrm{zu}$ keinen langatmigen, themenbezogen irrelevanten Verzögerungen kommt. Eine solche Klassenführung steht in einem positiven Zusammenhang zum Engagement der Schülerinnen und Schüler sowie in negativer Korrelation mit 
Unterrichtsstörungen (Kounin, 2006). Diese Variable lässt sich als Unterrichtsfluss beobachten, in dem der inhaltlichthematische Bogen durch die Vermeidung von Verzögerungen erhalten bleibt. Das Monitoring umschreibt eine Klassenführung, in der die Lehrkraft alle Ereignisse im Blick hat und dies den Schülerinnen und Schülern signalisiert (Casale, Strauß, Hennemann, \& König, 2016). Festgestellt wurde, dass ein gutes Monitoring, bei der die Lehrkraft die Gruppe als Ganzes im Blick hat ohne die einzelnen Schülerinnen und Schüler aus den Augen zu verlieren, die Mitarbeit der Lernenden erhöht und aversive Handlungen verringert (Gettinger \& Kohler, 2006; Kounin, 2006). Die latente Variable Überlappung charakterisiert die Fähigkeit des Multitaskings, die sich darin zeigt, dass die Lehrkraft in der Lage ist, auf mehrere handlungsauffordernde Situationen gleichzeitig reagieren $\mathrm{zu}$ können. Dabei kann es beispielsweise darum gehen, Verständnisschwierigkeiten, Ablaufprobleme oder aufkommende Sicherheitsproblematiken nebenbei zu beheben, ohne den Unterrichtsfokus zu verlieren. Aus den Forschungsbefunden geht hervor, dass ein guter Umgang mit überlappenden Ereignissen $\mathrm{zu}$ weniger aversiven Handlungen und $\mathrm{zu}$ einem höheren Engagement der Schülerinnen und Schüler führt (Kounin, 2006). Es geht dabei um die hohe Bedeutung einer adäquaten und unmittelbaren Reaktion auf gleichzeitig stattfindende Geschehnisse.

\section{Fragestellung und Methode}

Vor dem Hintergrund der in den vorhergehenden Abschnitten wird im vorliegenden Beitrag die folgende Fragestellung bearbeitet: Welche theoretisch abgeleiteten latenten (und manifesten) Variablen erweisen sich für die Erfassung klassenführungsbezogener Performanzen bei (angehenden) sportunterrichtenden Lehrkräften als inhaltlich sowie empirisch valide?

Um diese Fragestellung bearbeiten zu können, wurde eine dreistufige Vorgehensweise gewählt, in welcher sowohl qualitative als auch quantitative Verfahren zum Einsatz kamen (Mixed-meth-
ods-Vorgehensweise). Im Folgenden werden das Vorgehen, die Stichproben, das Raterinnen- und Ratertraining sowie das Datenanalysenverfahren dargestellt.

\section{Dreistufige Untersuchungs- durchführung}

In einem ersten Schritt wurden auf der Grundlage einer fachübergreifenden sowie fachspezifischen Literatur- und Instrumentenanalyse der Forschungsstand zur Klassenführung zusammengefasst und relevante latente Variablen sowie hochinferente manifeste Variablen $\mathrm{zu}$ einer guten Klassenführung im Fach Sport abgeleitet bzw. neu generiert (u.a. Cothran \& Kulinna, 2015; Heemsoth, 2014; Kounin, 2006; Piwowar, 2013). Das aus diesem Prozess resultierende Manuskript wurde im Projektteam in einem diskursiven Prozess, der mehrfach wiederholt wurde, überarbeitet und weiterentwickelt. Ziel dieses Vorgehens war die Erarbeitung eines Manuskripts, das den aktuellen Forschungsstand zusammenfasst und das vorläufige Beobachtungsinstrument darlegt. Das resultierende Beobachtungsinstrument bestand aus 10 latenten Variablen (s. Abschnitt „Qualitätsdimensionen einer guten Klassenführung im (Sport-)Unterricht") und 55 manifesten Variablen.

Zur inhaltlichen Validierung des Beobachtungsinstruments wurde in einem zweiten Schritt am Projektstandort der PH St.Gallen $(\mathrm{CH})$ eine Delphi-Runde (Heinzer \& Baumgartner, 2013) durchgeführt. Hierzu wurde den teilnehmenden 8 Expertinnen und Experten vor der Durchführung das vorliegende Manuskript zur Bearbeitung zugestellt. In der fünfstündigen Delphi-Runde wurden die Ziele des Forschungsprojekts vorgestellt, der aktuelle Forschungsstand dargelegt, die Variablen erläutert und offene Fragen geklärt. Folgend wurden die hergeleiteten Variablen in einem offenen Diskurs besprochen. Unklare manifeste Variablen wurden inhaltlich überarbeitet, redundante gestrichen und neue generiert. Das Beobachtungsinstrument bestand nach diesem Prozess aus 10 latenten und 40 manifesten Variablen.

Zur empirischen Validierung des Beobachtungsinstruments wurde in ei- nem dritten Schritt ein hochinferentes fremdbestimmtes Beurteilungsverfahren durchgeführt, d.h. die Qualität der klassenführungsbezogenen Performanzen einer sportunterrichtenden Lehrkraft wurde mittels des vorliegenden Beobachtungsinstruments durch wissenschaftliche Mitarbeitende und Hilfskräfte beurteilt. Die Qualität der Klassenführung wurde dabei anhand von 166 Unterrichtsstunden bei 91 angehenden und tätigen sportunterrichtenden Lehrkräften verschiedener Schulstufen (w. $=46 ; \mathrm{m} .=45$; Alter: $\mathrm{m}=37,48$, $\mathrm{SD}=12,79$, Range $=21-64$ Jahre) beurteilt. Da wir davon ausgehen, dass die dargelegten Variablen unabhängig von der Schulstufe bedeutsame Qualitätsdimensionen eines guten Sportunterrichts darstellen, wurden die Daten stufenübergreifend erhoben. Die Datengrundlage stellten zum einen 49 Unterrichtsvideos (à $45 \mathrm{~min}$ ) von 25 angehenden sportunterrichtende Lehrkräften der Berufsfachschule dar, welche als Lehrgang im Jahr 2013 an der Eidg. Hochschule für Sport Magglingen (EHSM; CH) ihre Ausbildung zur Sportlehrkraft absolvierten (w. $=8 ; \mathrm{m} .=17$; Alter: $\mathrm{m}=24,20$ Jahre, $\mathrm{SD}=2,90$; Range $=20-33$ Jahre). ${ }^{2}$ Zum anderen wurden 117 Unterrichtsstunden von 66 tätigen sportunterrichtenden Lehrkräften im Feld beurteilt (w. $=38$; m. $=28$; Alter: $\mathrm{m}=43,91, \mathrm{SD}=10,08$, Range $=26-64$ Jahre). Dazu wurden 29 Unterrichtsstunden von 17 sportunterrichtenden Lehrkräften der Primarstufe (w. $=15 ; \mathrm{m} .=2$; Alter: $\mathrm{m}=44,18$, $\mathrm{SD}=7,97$, Range $=35-60$ Jahre), 10 Unterrichtsstunden von 5 sportunterrichtenden Lehrkräften der Sekundarstufe I (w. $=3 ; \mathrm{m} .=2$; Alter: $\mathrm{m}=46,20$, $\mathrm{SD}=6,06$, Range $=38-54$ Jahre; jeweils Projektstandort der PH Ludwigsburg (D)) sowie 78 Unterrichtsstunden von 44 sportunterrichtenden Lehrkräfte der Sekundarstufe II (w. $=20$; m. $=24$; Alter: $\mathrm{m}=43,39, \quad \mathrm{SD}=11,47$, Range $=26-64$ Jahre; Projektstandort der PH St.Gallen) beobachtet. Folglich wurden die klassenführungsbezogenen Performanzen der (angehende) sport-

\footnotetext{
2 Diese Unterrichtsvideos wurden im Rahmen einer Interventionsstudie (Baumgartner, 2017b) generiert.
} 
unterrichtenden Lehrkräfte anhand des Beobachtungsinstruments teilweise doppelt beurteilt. Dies war notwendig, weil für die vorliegende Studie nicht genügend Schulen gewonnen werden konnten, um jede (angehende) sportunterrichtende Lehrkraft lediglich einmal zu beurteilen. Die Daten sind dementsprechend nicht unabhängig zu deuten. Weil die doppelt beurteilten (angehenden) Lehrkräfte jedoch jeweils unterschiedliche Inhalte in unterschiedlichen Klassen unterrichteten, wurden die Daten trotz der abhängigen Datenstruktur zur Validierung des Beobachtungsinstruments verwendet.

\section{Stichprobe der Expertinnen und Experten der Delphi-Runde sowie der Raterinnen und Rater zur empirischen Validierung des Beobachtungsinstruments}

Die Expertinnen und Experten des Faches Sport, die an der Delphi-Runde teilgenommen haben, wurden anhand der folgenden drei Kriterien ausgewählt: Sie mussten a) über ein Diplom als Lehrkraft verfügen bzw. das zweite Staatsexamen bestanden haben, $b$ ) als sportunterrichtende Lehrkraft arbeiten oder gearbeitet haben sowie c) in der Ausbildung tätig sein. Hierdurch sollte sichergestellt werden, dass die Variablen sowohl aus theoretischer als auch aus unterrichtspraktischer Perspektive beurteilt werden können (Baumgartner, 2018b). Es wurden 15 Fachdidaktikerinnen und Fachdidaktiker, die diese Kriterien erfüllen, angefragt, an der Delphi-Runde teilzunehmen, wovon sich zwei Fachdidaktikerinnen und sechs Fachdidaktiker beteiligten. Der Altersdurchschnitt lag bei 42,50 Jahren $(S D=7,69)$, die Altersspanne reichte von 34 bis 54 Jahren. Sie realisieren durchschnittlich seit 10,38 Jahren $(S D=5,26)$ hochschulischen Unterricht und lehren $7,5 \mathrm{~h}$ pro Woche $(\mathrm{SD}=4,54)$.

Hinsichtlich der empirischen Validierung des Beobachtungsinstruments konnten 4 wissenschaftliche Mitarbeitende sowie 2 wissenschaftliche Hilfskräfte als Raterinnen und Rater gewonnen werden ( $\mathrm{w} .=2 ; \mathrm{m} .=4)$. Der Altersdurchschnitt lag bei 30,17 Jahren $(\mathrm{SD}=4,49)$ und die Altersspanne reichte von 23-37 Jahren. Die wissenschaftlichen Mitarbeitenden verfügen alle über ein Lehrdiplom der Sekundarstufe II $(n=4)$, die wissenschaftlichen Hilfskräfte verfügen über einen BA Grundschullehramtsabschluss $(n=1)$ bzw. befanden sich in der Abschlussphase zum Erwerb des 1. Staatexamens für die Sekundarstufe I $(n=1)$ und haben bereits ihr Praxissemester absolviert.

\section{Raterinnen-/Ratertraining und Datenerhebung}

Eine notwendige Voraussetzung zur empirischen Validierung des Beobachtungsinstruments stellt eine hohe absolute Übereinstimmung bzw. eine hohe Interraterreliabilität der Ratenden dar. Dies bedingt, dass die Ratenden ein präzises Verständnis über die Qualitätskriterien (Variablen) sowie die Qualität der Merkmalsausprägungen hinsichtlich der manifesten Variablen (vierstufige Likert-Skala mit den verbalen Markern: $0=$,trifft nicht $z u^{\prime \prime} ; 1=$,trifft eher nicht $z u$ “; $2=$,trifft eher $z u$ “; $3=$ "trifft $\left.z u^{\prime \prime}\right)$ erlangen (Wirtz \& Caspar, 2002), was in vorliegender Studie durch ein Ratendentraining realisiert wurde. ${ }^{3} \mathrm{Im}$ Rahmen des Trainings wurde den Teilnehmenden in einem ersten Schritt vor der Durchführung des Trainings das Manuskript und das inhaltlich validierte Beobachtungsinstrument zur Verarbeitung zugestellt. Im Training, das ca. $4 \mathrm{~h}$ dauerte, wurden in einem zweiten Schritt die Ziele des Forschungsprojekts vorgestellt, die Variablen erläutert, die Merkmalsausprägungen thematisiert und offene Fragen geklärt. In einem dritten Schritt haben jeweils zwei Raterinnen und Rater unter Berücksichtigung des Beobachtungsinstruments ein erstes (stufenbezogenes) Unterrichtsvideo von (angehenden) sportunterrichtenden Lehrkräften in einem offenen Diskurs

\footnotetext{
3 Die empirische Validierung des Beobachtungsinstruments wurde an zwei Projektstandorten durchgeführt (PH St.Gallen $(\mathrm{CH})$; $\mathrm{PH}$ Ludwigsburg (D)). Dementsprechend wurde auch an beiden Projektstandorten jeweils ein Ratendentraining durchgeführt.
}

geratet. ${ }^{4}$ Die jeweiligen Urteilsabweichungen wurden innerhalb des Ratingteams besprochen und Fragen durch die Verantwortlichen der Projektstandorte geklärt. In einem vierten Schritt wurden in den Ratendenteams jeweils unabhängig voneinander zwei weitere Videos geratet, Urteilsabweichungen aufgegriffen und die Beurteilung vor dem Hintergrund des geführten Diskurses angepasst (Konsensurteil). In einem fünften Schritt wurden offene Fragen geklärt. Schritt vier und fünf fanden am Projektstandort der PH Ludwigsburg während der ersten Beobachtung im Feld statt.

Die 49 Unterrichtsvideos der angehenden sportunterrichtenden Lehrkräfte der Berufsfachschule wurden im Rahmen eines hochinferenten fremdbestimmte Beurteilungsverfahren am Projektstandort der PH St.Gallen durch eine Raterin und einen Rater unabhängig voneinander gescort („,double scoring“). Die Datenerhebung im Feld fand am Projektstandort der PH St.Gallen an drei Berufsfachschulen und zwei Gymnasien statt. Die Datenerhebung im Feld am Projektstandort der PH Ludwigsburg fand an zwei Grundschulen und einer Sekundarschule I statt. Die Ratenden beurteilten die klassenführungsbezogenen Performanzen der videografierten angehenden und der unterrichtenden Lehrkräfte während der Beobachtung und setzten am Ende der Lektion die jeweiligen variablenbezogenen Qualitätsausprägungen. Um die Güte der Beurteilung der Ratenden quantifizieren zu können, wurden die 49 Unterrichtsvideos sowie 12 Unterrichtsstunden im Feld jeweils durch zwei Ratende beurteilt („double scoring“). Die personenbezogenen Daten der Lehrkräfte wurden nach der Stunde mittels Fragebogen erhoben. Hinsichtlich der Videografie wurde das Einverständnis der Schulleitung mündlich sowie der angehenden sportunterrichtenden Lehrkräfte und

\footnotetext{
4 Im Ratendentraining des Projektstandorts der PH Ludwigsburg wurde ein Unterrichtsvideo der Jahrgangstufe fünf verwendet, im Training des Projektstandorts der PH St.Gallen wurden hierbei stufenbezogen auf die Unterrichtsvideos der angehenden sportunterrichtenden Lehrkräfte derBerufsfachschule zurückgegriffen.
} 


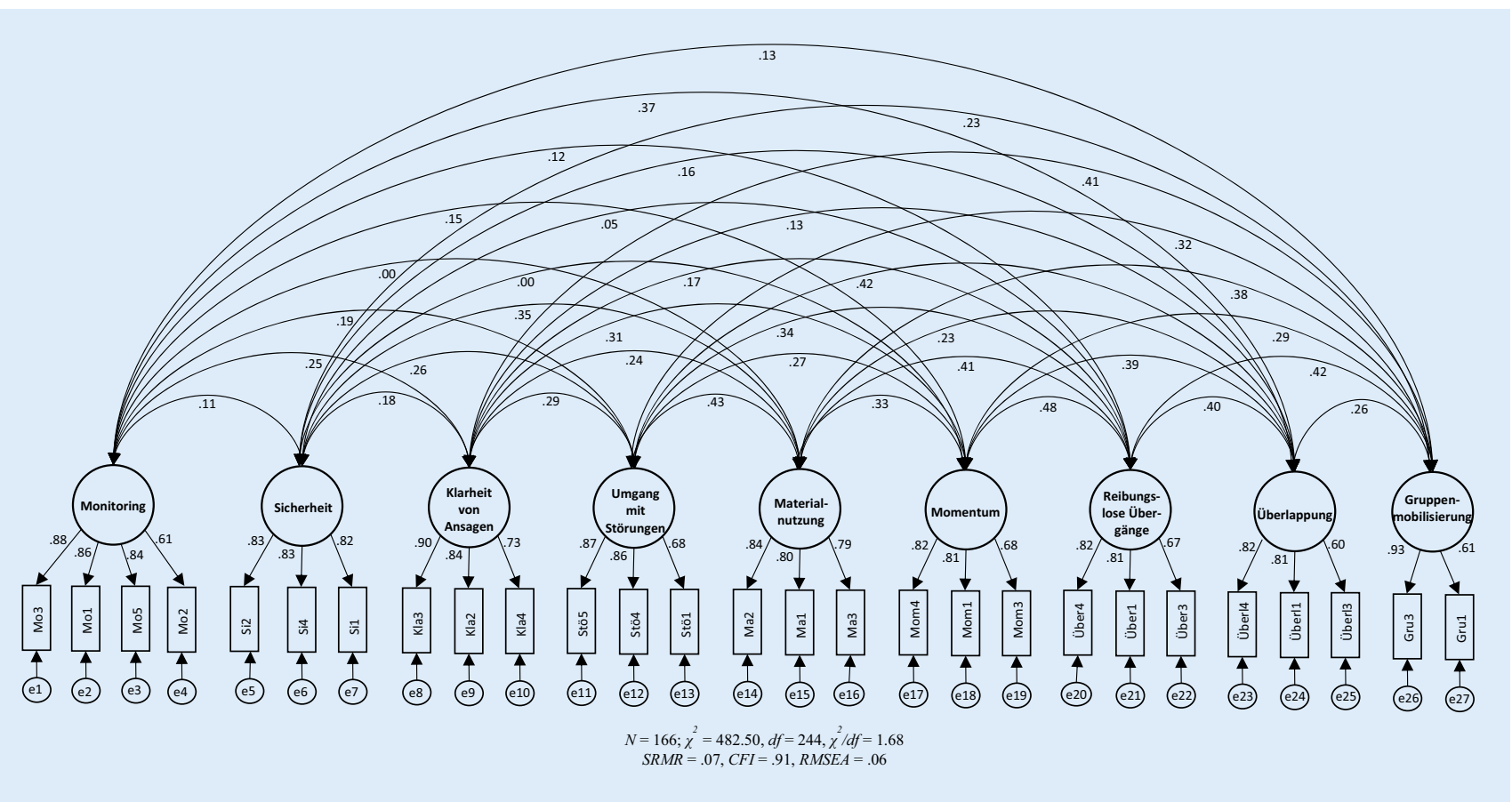

Abb. 1 A Konstrukt klassenführungsbezogene Performanzen im Fach Sport - 9-faktorielles Messmodell - konfirmatorische Faktorenanalyse

der Schülerinnen und Schüler schriftlich eingeholt. In den Beobachtungen im Feld wurde das Einverständnis der Schulleitung, der Lehrkräfte und der Schülerinnen und Schüler mündlich eingeholt.

\section{Datenanalyseverfahren}

Um die absolute Übereinstimmung der Ratendenurteile des Beobachtungsverfahrens quantifizieren zu können, wurden die prozentuale Übereinstimmung $(P)$ und das Übereinstimmungsmaß Cohens Kappa (К) berechnet. Die Güte der Interraterreliabilität wurde anhand des Reliabilitätskoeffizienten ICC eruiert. Zur Überprüfung der Qualität des postulierten reflexiven Modells im Sinne eines hypothetischen Konstrukts wurde das strukturentdeckende Verfahren der explorativen Faktorenanalyse bzw. die Hauptkomponentenanalyse (HKA) mit Promax-Rotation durchgeführt (IBM SPSS 23). Auf der Grundlage einer Inter-Item-Korrelation wird mit diesem multivariaten Verfahren überprüft, welche Qualität die Zusammenhänge zwischen den manifesten und den latenten Variablen aufweisen. Es wurde die HKA angewandt, weil durch dieses datenreduzierende Verfahren ein möglichst ökonomisches Beobachtungsinstrument generiert werden soll (Bühner, 2011). Diesbezüglich wurden manifeste Variablen ausgeschlossen, welche eine geringe Faktorenladung $(\lambda<0,50)$, einen geringen Kommunalitätswert $\left(h^{2}<0,60\right)$ oder einen geringen korrigierten Trennschärfekoeffizient $\quad\left(\mathrm{r}_{\text {itt }(\mathrm{i})}<0,30\right) \quad$ zeigten (Bühner, 2011). Zur Quantifizierung der Güte der latenten Variablen wurde der Kaiser-Mayer-Olkin-Test (KMO-Test) sowie der Barlett-Test durchgeführt. Die interne Konsistenz wurde mit der Berechnung des Cronbachs Alpha (a) quantifiziert. Die empirische Extraktion der latenten Variablen wurde in Anlehnung an das Kaiser-GutmannKriterium realisiert. Zur Überprüfung der Qualität des reflexiven Modells wurde das strukturüberprüfende Verfahren der konfirmatorischen Faktorenanalyse (KFA) durchgeführt (IBM SPSS Amos 23, New York). Dabei sollte nach MacCallum und Widaman (1999) das Verhältnis der Items zur Stichprobengröße 1:5 nicht unterschritten werden, was in vorliegender Studie eingehalten werden kann. Die Güte des Messmodells wurde mittels
Maximum-Likelihood-Methode (ML) geschätzt. Im ML-verfahren werden die Populationsparameter der vorliegenden Datenstruktur geschätzt bzw. es wird die Wahrscheinlichkeit überprüft, ob das Modell in einer Grundgesamtheit gilt (Rost, 2004). Diesbezüglich wurde die Bootstrap-Methode mit 1000 Iterationen angewandt, d.h. es wurde in Bezug auf das proklamierte Messmodell 1000mal weitere Stichproben gezogen, um das Modell zu überprüfen (Chernick, 2008). Fehlende Werte wurden mit der FIML-Technik bearbeitet und dadurch direkt in der Parametereinschätzung ersetzt (Weiber \& Mühlhaus, 2010). Zur Bewertung der Güte des Modells wurde die Fit-Indizes (RMSEA, CFI, SRMR, $\chi^{2} / \mathrm{df}$ ) berechnet. $^{5} \mathrm{Um}$ die Reliabilität

\footnotetext{
${ }^{5}$ Nach Browne und Cudeck (1993) ist ein RMSEA-Wert von $\leq 0,05$ als gut und $\geq 0,10$ als inakzeptablen zu interpretieren. Ein ausreichender $\mathrm{CFI}$-Wert ist eine Ausprägung von $>0,90$ (Bentler, 1990). Der SRMR-Wert von $<0,05$ ist als gut (Homburg, Klarmann, \& Pflessner, 2008) bzw. der Wert von $\leq 0,10$ (Backhaus, Erichson, \& Weiber, 2013) als akzeptabel zu deuten. Der Modell-Fit mit einem Verhältnis von $x^{2} / d f<3$ ist als gut bzw. $x^{2} / \mathrm{df}<2$ als sehr gut zu bewerten (Byrne, 2010).
} 
Tab. 1 Faktorladungen und Kommunalitätswerte der explorativen Faktorenanalyse (HKA mit Promax-Rotation)

\begin{tabular}{|c|c|c|c|c|c|c|c|c|c|}
\hline & Faktor & & & & & & & & \\
\hline Itemcode & 1 & 2 & 3 & 4 & 5 & 6 & 7 & 8 & 9 \\
\hline Mo3 & $0,92(0,83)$ & - & - & - & - & - & - & - & - \\
\hline Mo1 & $0,91(0,82)$ & - & - & - & - & - & - & - & - \\
\hline Mo5 & $0,84(0,79)$ & - & - & - & - & - & - & - & - \\
\hline Mo2 & $0,69(0,60)$ & - & - & - & - & - & - & - & - \\
\hline $\mathrm{Si} 2$ & - & $0,92(0,81)$ & - & - & - & - & - & - & - \\
\hline $\mathrm{Si} 4$ & - & $0,90(0,80)$ & - & - & - & - & - & - & - \\
\hline Si1 & - & $0,87(0,82)$ & - & - & - & - & - & - & - \\
\hline Kla4 & - & - & $0,91(0,78)$ & - & - & - & - & - & - \\
\hline Kla3 & - & - & $0,86(0,85)$ & - & - & - & - & - & - \\
\hline Kla2 & - & - & $0,83(0,79)$ & - & - & - & - & - & - \\
\hline Stö5 & - & - & - & $0,97(0,86)$ & - & - & - & - & - \\
\hline Stö4 & - & - & - & $0,91(0,83)$ & - & - & - & - & - \\
\hline Stö1 & - & - & - & $0,73(0,67)$ & - & - & - & - & - \\
\hline Ma2 & - & - & - & - & $0,97(0,85)$ & - & - & - & - \\
\hline Ma1 & - & - & - & - & $0,88(0,81)$ & - & - & - & - \\
\hline Ma3 & - & - & - & - & $0,75(0,77)$ & - & - & - & - \\
\hline Mome3 & - & - & - & - & - & $0,90(0,74)$ & - & - & - \\
\hline Mome1 & - & - & - & - & - & $0,86(0,77)$ & - & - & - \\
\hline Mome4 & - & - & - & - & - & $0,80(0,77)$ & - & - & - \\
\hline Über3 & - & - & - & - & - & - & $0,88(0,76)$ & - & - \\
\hline Über4 & - & - & - & - & - & - & $0,82(0,76)$ & - & - \\
\hline Über1 & - & - & - & - & - & - & $0,76(0,75)$ & - & - \\
\hline Überla3 & - & - & - & - & - & - & - & $0,87(0,76)$ & - \\
\hline Überla4 & - & - & - & - & - & - & - & $0,74(0,76)$ & - \\
\hline Überla1 & - & - & - & - & - & - & - & $0,74(0,72)$ & - \\
\hline Gru1 & - & - & - & - & - & - & - & - & $0,93(0,85)$ \\
\hline Gru3 & - & - & - & - & - & - & - & - & $0,80(0,79)$ \\
\hline
\end{tabular}

der Konstruktmessung quantifizieren $\mathrm{zu}$ können, werden die Faktorreliabilitäten (Rel) berichtet. ${ }^{6}$

\section{Darstellung der Ergebnisse}

Hinsichtlich der empirischen Validierung wurde in einem ersten Schritt die HKA auf mehrfaktorieller Ebene durchgeführt. Die latente Variable Performanztransparenz musste dabei ausgeschlossen werden, weil diese Dimension im Sportunterricht nur bei 33,32\% der Beobachtungen beurteilt wurde und die Variable aufgrund des hohen Anteils

${ }^{6}$ Nach Bagozzi und Yi (1988) gilt ein Wert von $>0,60$ für eine reliable Konstruktmessung. an fehlenden Werten $(N>10 \%$; Weiber \& Mühlhaus, 2010) zur Berechnungen nicht weiterverwendet werden konnte. Aufgrund einer zu geringen Faktorenladung $(\lambda<0,50)$, einem $\mathrm{zu}$ geringen Kommunalitätswert $\left(h^{2}<0,60\right)$ oder einem zu geringen Trennschärfekoeffizient $\left(r_{i t(i)}<0,30\right)$ musste von den inhaltlich validierten 40 manifesten Variablen 13 ausgeschlossen werden. Der Anteil fehlender Werte bezüglich der validierten 27 manifesten Variablen ist gering (4,8\%; Weiber \& Mühlhaus, 2010), weshalb die Daten zur weiteren Berechnung verwendet werden konnten. Aus der HKA geht eine 9-faktorielle Lösung hervor (vgl. - Tab. 1). Die 9 latenten Variablen erklären insgesamt 78,14\% der
Gesamtvarianz. Die Kennwerte sind als zufriedenstellend bis gut $\mathrm{zu}$ bezeichnen $(\mathrm{KMO}=0,78$, Barlett $=0,000, \alpha=0,87)$. Die verbleibenden 27 manifesten Variablen weisen gute Faktorladungen $(0,69 \leq \lambda \leq 0,97)$ sowie befriedigende Kommunalitätswerte $\quad\left(0,60 \leq \mathrm{h}^{2} \leq 0,86\right)$ auf.

Zur Überprüfung des Messmodells wurde die konfirmatorische Faktorenanalyse (KFA) durchgeführt. Die Fit-Indizes der 9-faktoriellen Lösung weisen auf akzeptable bis hohe Güte hin $\left(N=166 ; \chi^{2}=482,50, \mathrm{df}=288\right.$, $\chi^{2} / \mathrm{df}=1,68, \mathrm{SRMR}=0,07, \mathrm{CFI}=0,91$, RMSEA $=0,06$ ). Damit konnte das proklamierte Modell auf der Grundlage der vorliegenden Daten validiert werden (vgl. 


\begin{tabular}{|c|c|c|c|c|c|c|}
\hline $\begin{array}{l}\text { Latente Va- } \\
\text { riablen }\end{array}$ & $\begin{array}{l}\text { Anzahl mani- } \\
\text { feste Varia- } \\
\text { blen }\end{array}$ & $\mathbf{M}$ & SD & a & Rel & Beispielitem \\
\hline Monitoring & 4 & 2,22 & 0,63 & 0,88 & 0,88 & $\begin{array}{l}\text { Die sportunterrichtende Lehrkraft wählt Positionierungen im Raum, } \\
\text { von denen aus sie einen guten Überblick über das Klassengeschehen } \\
\text { hat }\end{array}$ \\
\hline Sicherheit & 3 & 2,63 & 0,57 & 0,86 & 0,87 & $\begin{array}{l}\text { Die sportunterrichtende Lehrkraft gestaltet die Lernumgebung } \\
\text { sicher (z. B. Verwendung von Weichboden etc.) }\end{array}$ \\
\hline $\begin{array}{l}\text { Klarheit von } \\
\text { Ansagen }\end{array}$ & 3 & 2,25 & 0,67 & 0,86 & 0,87 & $\begin{array}{l}\text { Die sportunterrichtende Lehrkraft formuliert Ziele/Aufgaben/ } \\
\text { Übungen verständlich }\end{array}$ \\
\hline $\begin{array}{l}\text { Umgang mit } \\
\text { Störungen }\end{array}$ & 3 & 2,24 & 0,64 & 0,84 & 0,85 & $\begin{array}{l}\text { Die sportunterrichtende Lehrkraft klärt kleinere Störungen, ohne } \\
\text { den Unterrichtsfluss zu unterbrechen }\end{array}$ \\
\hline $\begin{array}{l}\text { Material- } \\
\text { nutzung }\end{array}$ & 3 & 2,60 & 0,54 & 0,84 & 0,85 & $\begin{array}{l}\text { Die im Sportunterricht verwendeten Großgeräte (z. B. Barren), Klein- } \\
\text { geräte (z. B. Bälle) und Unterrichtsmaterialien (z. B. Videos, Flipcharts } \\
\text { etc.) unterstützen den Lernprozess der Lernenden }\end{array}$ \\
\hline Momentum & 3 & 2,44 & 0,58 & 0,82 & 0,82 & $\begin{array}{l}\text { Die sportunterrichtende Lehrkraft ist während den Erklärungen nicht } \\
\text { weitschweifig }\end{array}$ \\
\hline $\begin{array}{l}\text { Reibungslose } \\
\text { Übergänge }\end{array}$ & 3 & 2,21 & 0,64 & 0,82 & 0,83 & $\begin{array}{l}\text { Die sportunterrichtende Lehrkraft organisiert die Übergänge so, dass } \\
\text { der Unterricht ohne größere Unterbrechungen weiterlaufen kann }\end{array}$ \\
\hline Überlappung & 3 & 2,00 & 0,62 & 0,78 & 0,79 & $\begin{array}{l}\text { Die sportunterrichtende Lehrkraft kann sich einem Unterrichtsereig- } \\
\text { nis widmen, ohne die Klasse zu vernachlässigen }\end{array}$ \\
\hline $\begin{array}{l}\text { Gruppen- } \\
\text { mobilisierung }\end{array}$ & 2 & 2,38 & 0,69 & - & - & $\begin{array}{l}\text { Die sportunterrichtende Lehrkraft achtet darauf, dass sich möglichst } \\
\text { alle Lernende mit dem Lerngegenstand befassen }\end{array}$ \\
\hline
\end{tabular}

- Abb. 1). Aufgrund der geringen Faktorinterkorrelationen $\quad(0,00 \leq \Phi \leq 0,48)$ wird von einer Überprüfung eines Messmodells zweiter Ordnung abgesehen, d.h. es kann keine latente Variable zweiter Ordnung gebildet werden, der mehrere latente Variablen erster Ordnung (z. B. Monitoring) subsumiert werden können. ${ }^{7}$

Um die Güte der Urteile der Ratenden quantifizieren zu können, wurden die absolute Übereinstimmung $(P, \mathrm{~K})$ bzw. die Interraterreliabilität (ICC einfach, zuf.) bezüglich der empirisch validierten $27 \mathrm{ma}$ nifesten Variablen berechnet. Dabei wurden 49 Unterrichtsvideos sowie 12 Unterrichtsstunden in vivo doppelt gescort. Die Ergebnisse weisen auf eine gute bis exzellente Raterübereinstimmung bzw. Raterreliabilität $(P=0,69 ; \mathrm{K}=0,50$; $\mathrm{ICC}_{\text {einfach, } \text { zuf.. }}=0,80 ; \mathrm{F}[1,1647]=4,77$, $p<0,001)$. Zusammenfassend kann festgehalten werden, dass mit dem vorliegenden Datensatz ein multidimensionales,

\footnotetext{
7 Nach Brown (2006) soll ein Modell zweiter Ordnung dann geprüft werden, wenn eine Korrelation zwischen zwei latenten Variablen eine Ausprägung von $>0,50$ aufweist.
}

9-faktorielles Beobachtungsinstrument zur Erfassung der klassenführungsbezogenen Performanzen von sportunterrichtenden Lehrkräften validiert werden konnte.

Aus den Ergebnissen lassen sich unter Berücksichtigung der empirischen Lösung (vgl. @ Abb. 1) die postulierten 9 latenten Variablen extrahieren. Das arithmetische Mittel (M) der validierten latenten Variablen bzw. der manifesten Variablen liegt zwischen 2,00 und $2,63(0,54 \leq \mathrm{SD} \leq 0,69)$. Das Cronbachs Alpha $(0,78 \leq \alpha \leq 0,88)$ sowie die Faktorreliabilität $(0,79 \leq \mathrm{Rel} \leq 0,88)$ weisen auf zufriedenstellende Koeffizientwerte (vgl. - Tab. 2).

\section{Diskussion}

\section{Beantwortung der Fragestellung}

In der vorliegenden Studie wurde die Entwicklung und die empirische Validierung eines multidimensionalen Beobachtungsinstruments zur Erfassung der Qualität der klassenführungsbezogenen Performanzen von sportunterrichtenden Lehrkräften dargelegt (KlaPe-Sport). Vor dem Hintergrund der fachübergreifenden und fachspezifischen Forschungsbeiträge, aus der Realisierung einer Delphi-Runde sowie der empirischen Überprüfung des generierten Modells ging ein 9-faktorielles reflexives Messmodell hervor, das aus 27 manifesten Variablen besteht. Die 9-faktorielle Lösung weist einen ausreichenden bis guten Modell-Fit auf. Die Berechnung der absoluten Raterinnen- und Raterübereinstimmung sowie der Interraterreliabilität zeigten gute bis exzellente Kennwerte. Dementsprechend kann festgehalten werden, dass die faktorielle Validität sowie die Reliabilität des KlaPe-Sport-Beobachtungsinstruments eine ausreichende bis hohe Güte aufweisen.

Das validierte Modell unterscheidet sich dabei von anderen Rahmenmodellen zum hier thematisierten Bereich der Qualität der Klassenführung. So legt Piwowar (2013) ein fachübergreifendes Beobachtungsinstrument vor, welches aus einer Struktur zweiter Ordnung besteht. Dabei werden verschiedene latente Variablen den drei Variablen zweiter Ordnung Management der Instruktion, Management von Schülerinnen- und 
Schülerverhalten, Management der Lehrer-Schüler-Beziehung subsumiert. Für das vorliegende fachspezifische Beobachtungsinstrument ergibt sich hingegen eine Lösung erster Ordnung, weil aus Daten eine Struktur zweiter Ordnung nicht generiert werden kann. Neben den fachspezifischen Unterschieden werden außerdem, im Vergleich $\mathrm{zu}$ dem auf Instruktion und Regulierung des Schülerverhaltens basierenden Modell von Piwowar (ebd.), Gestaltungsaspekte unterstützender Lehr-Lernsituationen stärker berücksichtigt. In jüngster Zeit haben auch Linka und Gerlach (2019) im Kontext der Erstellung von Selbsteinschätzungsbögen zum Klassenmanagement im Sportunterricht aus Lehrer-I Lehrerinnen- sowie Schüler- und Schülerinnensicht ein Rahmenmodell zum Klassenmanagement vorgelegt, dessen empirische Überprüfung bislang noch aussteht. ${ }^{8}$ Auch in diesem fachspezifischen Zugang basiert die Strukturierung des Konstrukts, wie auch bei Piwowar (2013), auf einem stärker lehrerorientierten Fokus. Das vorgeschlagene Rahmenmodell richtet sich zudem auf den weiter gefassten Begriff des Klassenmanagements (vgl. Abschnitt „Einleitung“). In Abgrenzung dazu wurde in vorliegender Studie ein Instrument generiert, womit die beobachtbare Klassenführung, d.h. die diesbezüglichen Performanzen von Lehrkräften, erfasst werden kann.

\section{Limitationen}

Die empirische Validierung des Beobachtungsinstruments wurde in der vorliegenden Studie stufenübergreifend realisiert. Es kann davon ausgegangen werden, dass die generierten Merkmale einer guten Klassenführung im Fach Sport in allen Schulstufen eine hohe Relevanz aufweisen. Ein stufenbezogener Vergleich des Messmodells steht allerdings noch aus, um diese Annahme zu stützen. Bedeutsam scheint außerdem eine weiterführende Überprüfung des Instruments

${ }^{8}$ Die vorliegende Untersuchung sowie die Studie von Linka und Gerlach (2019) verliefen zeitlich parallel. Daher konnte die Studie in dieser Arbeit erst gegen Ende und nicht bereits im theoretischen Teil berücksichtigt werden. hinsichtlich seiner Eignung, die Qualität von Klassenführung auch bei differenten methodisch-didaktischen Zugängen zu erfassen. Ebenfalls eine zukünftige Betrachtung verdient der Umstand, dass einelatenten Variablen (Performanztransparenz) trotz inhaltlicher Validität in der empirischen Überprüfung ausgeschlossen werden musste. In methodischer Hinsicht ist erwähnenswert, dass die vorliegende Studie auf einem abhängigen Datensatz basiert, d.h. (angehende) sportunterrichtenden Lehrkräfte wurden doppelt gescort. Da die doppelt beobachteten (angehenden) sportunterrichtenden Lehrkräfte jedoch in unterschiedlichen Klassen verschiedene Inhalte unterrichteten, wurden die Daten trotz der abhängigen Datenstruktur verwendet.

\section{Fazit und Ausblick}

Das validierte Beobachtungsinstrument KlaPe-Sport stellt eine Grundlage dar, um die Qualität der klassenführungsbezogenen Performanzen von (angehenden) sportunterrichtenden Lehrkräften erfassen zu können. Im Konkreten wird dadurch ermöglicht, dass die Qualität der Klassenführung im Sportunterricht anhand der instrumentbezogenen manifesten Variablen und den diesbezüglichen Merkmalsausprägungen beurteilt wird. So lassen sich mit dem Instrument beispielsweise Studien durchführen, welche die Effektivität von hochschuldidaktischen Lehr- und Lernarrangements hinsichtlich des Performanzfortschritts bei (angehenden) sportunterrichtenden Lehrkräften untersuchen. Des Weiteren erscheinen vor dem Hintergrund eines integrativen Kompetenzverständnisses (Baumgartner, 2018a) Untersuchungen erstrebenswert, mithilfe derer der $\mathrm{Zu}$ sammenhang zwischen der Qualität des klassenführungsbezogenen Wissens, der Wahrnehmungs-, Interpretationsund Entscheidungsfähigkeiten sowie der Qualität der Performanz erhellt wird. Auch hierbei kann das entwickelte Beobachtungsinstrument einen wichtigen Beitrag leisten, indem es im Vergleich zu Selbsteinschätzungsverfahren eine auf intersubjektiven Fremdbeurteilungsverfahren beruhende, ökologisch validere Erhebung der Performanz ermöglicht.

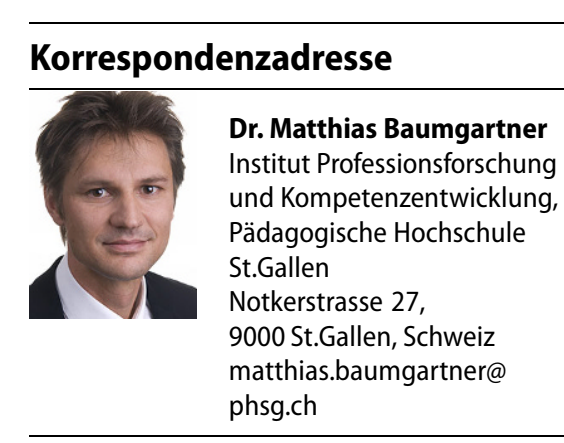

Funding. Open access funding provided by St.Gallen University of Teacher Education

\section{Einhaltung ethischer Richtlinien}

Interessenkonflikt. M. Baumgartner, V. Oesterhelt und S. Reuker geben an, dass kein Interessenkonflikt besteht.

Für diesen Beitrag wurden von den Autoren keine Studien an Menschen oder Tieren durchgeführt. Für die aufgeführten Studien gelten die jeweils dort angegebenen ethischen Richtlinien.

Open Access Dieser Artikel wird unter der Creative Commons Namensnennung 4.0 International Lizenz veröffentlicht, welche die Nutzung, Vervielfältigung, Bearbeitung, Verbreitung und Wiedergabe in jeglichem Medium und Format erlaubt, sofern Sie den/die ursprünglichen Autor(en) und die Quelle ordnungsgemäß nennen, einen Link zur Creative Commons Lizenz beifügen und angeben, ob Änderungen vorgenommen wurden.

Die in diesem Artikel enthaltenen Bilder und sonstiges Drittmaterial unterliegen ebenfalls der genannten Creative Commons Lizenz, sofern sich aus der Abbildungslegende nichts anderes ergibt. Sofern das betreffende Material nicht unter der genannten Creative Commons Lizenz steht und die betreffende Handlung nicht nach gesetzlichen Vorschriften erlaubt ist, ist für die oben aufgeführten Weiterverwendungen des Materials die Einwilligung des jeweiligen Rechteinhabers einzuholen.

Weitere Details zur Lizenz entnehmen Sie bitte der Lizenzinformation auf http://creativecommons.org/ licenses/by/4.0/deed.de.

\section{Literatur}

Backhaus, K., Erichson, B., \& Weiber, R. (2013). Fortgeschrittene Multivariate Analysemethoden: Eine anwendungsorientierte Einführung (2. Aufl.) Berlin:Springer.

Bagozzi, R., \& Yi, Y. (1988). On the evaluation of structural equation models. Journal of the Academy of Marketing Science, 16(1), 74-94.

Baumberger, J. (2018). Kompetenzorientierter Sportunterricht. Eine explorative Studie an Primarschulen zur Umsetzung des Lehrplans 21 Bewegung und Sport. Aachen: Meyer \& Meyer.

Baumert, J., \& Kunter, M. (2006). Stichwort: Professionelle Kompetenz von Lehrkräften. Zeitschrift für Erziehungswissenschaft, 9(4), 469-520. 
Baumgartner, M. (2013a). Kompetenzprofile von Sportlehrpersonen der Berufsfachschule. In F. Oser, T. Bauder, P. Salzmann \& S. Heinzer (Hrsg.), Ohne Kompetenz keine Qualität. Entwickeln und Einschätzen von Kompetenzprofilen bei Lehrpersonen und Berufsbildungsverantwortlichen (S. 96-126). Bad Heilbrunn: Klinkhardt.

Baumgartner, M. (2013b). Professionelle Kompetenzprofile von Sportlehrpersonen. Zur empirischen Generierung eines kompetenzorientierten Referenzsystems für die Ausbildung von Sportlehrpersonen der Berufsfachschule. Spectrum der Sportwissenschaften, 25(2), 49-74.

Baumgartner, M. (2017a). „Denn sie wissen nicht, was sie können...!" - die Qualität der Performanzen von angehenden Sportlehrkräften als Verzerrer der Selbstbeurteilung. German Journal of Exercise and Sport Research, 47(3), 246-254.

Baumgartner, M. (2017b). Performanzentwicklung in der Ausbildung von Lehrkräften. Eine Interventionsstudie zur Verbesserung des Feedbacks bei angehenden Sportlehrkräften. Münster: Waxmann.

Baumgartner, M. (2018a). „.... Kompetenz ohne Performanz ist leer! Performanz ohne Kompetenz blind...!" Zu einem integrativen Kompetenzstrukturmodell von Sportlehrkräften. Zeitschrift für sportpädagogische Forschung, 6(1), 49-68.

Baumgartner, M. (2018b). Performanzfortschritt in der Lehrerausbildung durch die Arbeit an eigenen video- und textbasierten Unterrichtsfällen? Eine Interventionsstudie zur Verbesserung des Feedbacks bei angehenden Sportlehrkräften. Zeitschrift für Erziehungswissenschaft, 21(6), 1135-1155.

Baumgartner, M., Owassapian, D., \& Perret Guldimann, J. (angenommen). Zur Verbesserung der Klassenführung von sportunterrichtenden Lehrkräfen auf der E-Learning-Plattform bewegunglesen.ch. Sportunterricht.

Bentler, P.M. (1990). Comparative fit indices in structural models. Psychological Bulletin, 107(2), 238-246.

Bohl, T., \& Kucharz, D. (2010). Offener Unterricht heute: konzeptionelle und didaktische Weiterentwicklung. Weinheim: Beltz.

Brouwers, A., \& Tomic, W. (2000). A longitudinal study of teacher burnout and perceived self-efficacy in classroom management. Teaching and Teacher Education, 16(2), 239-253.

Brown, T. A. (2006). Confirmatory factor analysis for applied research. New York: The Guilford Press.

Browne, M.W., \& Cudeck, R. (1993). Alternative ways of assessing model fit. In K.A. Bollen \& J.S. Long (Hrsg.), Testing structural equation models (S. 136-162). Beverly Hills: SAGE.

Bühner, M. (2011). Einführung in die Test- und Fragebogenkonstruktion (3. Aufl.). München:Pearson.

Byrne, B. (2010). Structoral equation modeling with AMOS. Basis concepts, application, and programming (2. Aufl.). New York: Routledge.

Casale, G., Strauß, S., Hennemann, T., \& König, J. (2016). Wie lässt sich Klassenführungsexpertise messen? Überprüfung eines videobasierten Erhebungsinstruments für Lehrkräfte unter Anwendung der Generalisierbarkeitstheorie. Empirische Sonderpädagogik, 8(2), 119-139.

Chepyator-Thomson, J.R., \& Liu, W. (2003). Preservice teachers' reflections on student teaching experiences: lessons learned and suggestions for reform in PETE programs. Physical Educator, $60(2), 2-12$.

Chernick, M. R. (2008). Bootstrap methods: a guide for practioners and researchers. New York: Wiley.
Cothran, D. J., \& Kulinna, P. H. (2015). Classroom management in physical education. In E. T. Emme \& E.J. Sabornie (Hrsg.), Handbook of classroom management (S. 239-260). New York: Routledge.

Dicke, T., Elling, J., Schmeck, A., \& Leutner, D. (2015). Reducing reality shock: the effects of classroom management skills training on beginning teachers. Teaching and Teacher Education, 48(1) 1-12.

Emmer, E. T., Evertson, C. M., \& Worsham, M. E. (2003). Classroom management for secondary teachers. Boston: Allyn und Bacon.

Fendick, F. (1990). The correlation between teacher clarity of communication and student achievement gain. A meta-analysis. Florida: University of Florida.

Funke-Wieneke, J. (2007). Grundlagen der Bewegungs und Sportdidaktik. Baltmannsweiler: Schneider.

Garrahy, D. A., Cothran, D. J., \& Kulinna, P.H. (2005). Voices from the trenches: an exploration of teachers' management knowledge. Journal of Educational Research, 99(1), 56-63.

Gettinger, M., \& Kohler, K. (2006). Process-outcome approaches to classroom management and effective teaching. In C. Evertson \& C. Weinstein (Hrsg.) Handbook of classroom management: research, practice, and contemporary issues (S. 73-96). Mahwah:Erlbaum.

Haag, L. (2018). Kernkompetenz Klassenführung. Heilbrunn: Klinkhardt.

Hattie, J. (2009). Visible learning: a synthesis of over 800 meta-analyses relating to achievement. London: Routledge.

Heemsoth, T. (2014). Unterrichtsklima als Mediator des Zusammenhangs von Klassenführung und Motivation im Sportunterricht. Psychologie in Erziehung und Unterricht, 61(3), 203-215.

Heinzer, S., \& Baumgartner, M. (2013). Bottom-up zur Qualität: Eine Quasi-Delphi-Studie zur Generierung von Kompetenzprofilen.InF.Oser, T.Bauder, P. Salzmann \& S. Heinzer (Hrsg.), Ohne Kompetenz keine Qualität. Entwickeln und Einschätzen von Kompetenzprofilen bei Lehrpersonen und Berufsbildungsverantwortlichen (S. 66-95). Bad Heilbrunn: Klinkhardt.

Helmke, A. (2017). Unterrichtsqualität und Lehrerprofessionalität. Diagnose, Evaluation und Verbesserung des Unterrichts. Seelze: Klett.

Herrmann, C., Seiler, S., \& Niederkofler, B. (2016). „Was ist guter Sportunterricht?" - Dimensionen der Unterrichtsqualität. Sportunterricht, 65(3), 7-12.

Hill, G., \& Brodin, K. L. (2004). Physical Education teachers' perceptions of the adequacy of university coursework in preparation for teaching. Physical Educator, 61(2), 75-87.

Hines, C., Cruickshank, D. R., \& Kennedy, J. J. (1985). Teacher clarity and its relationship to student achievement and satisfaction. American Educational Research Journal, 22(1), 87-99.

Homburg, C., Klarmann, M., \& Pflessner, C. (2008). Konfirmatorische Faktorenanalyse. In A. Herrmann, C. Homburg \& M. Klarmann (Hrsg.), Handbuch Marktforschung: Methoden, Anwendungen, Praxisbeispiele(S.271-303). Wiesbaden: Gabler.

Klinge, A. (2009). Die Scham istnie vorbei! Beschämung im Schulsport - eine sportpädagogische Herausforderung. Sportunterricht, 58(10), 296-301.

Kounin, J.S. (2006). Techniken der Klassenführung. Münster:Waxmann.

Kulinna, P.H., Silverman, S., \& Keating, X.D. (2000). Relationship between teachers' belief systems and actions toward teaching physical activity and fitness. Journal of Teaching in Physical Education, 19(2), 206-221.
Lavay, B., Henderson, H., French, R., \& Guthrie, S. (2012). Behavior management instructional practices and content of college/university physical education teacher education programs. Physical Education and Sport Pedagogy, 17(2), 195-210.

Linka, T., \& Gerlach, E. (2019). Klassenmanagement im Sportunterricht - Ergebnisse zur Evaluation der ersten Phase eines videogestützten Seminarkonzepts für angehende Lehrkräfte. In M. Hartmann, R. Laging \& C. Scheinert (Hrsg.), Professionalisierung in der Sportlehrer*innenbildung. Konzepte und Forschungen im Rahmen der "Qualitätsoffensive Lehrerbildung" (S.97-107). Hohengehren:Schneider.

MacCallum, R. C., \& Widaman, K. F. (1999). Sample size in factor analysis. Psychological Methods, 4(1), 84-99.

Miethling, W.-D., \& Krieger, C. (2004). Schüler im Sportunterricht. Schorndorf: Hofmann.

Oesterhelt, V., Gerlach, E., Grimminger-Seidensticker, E., \& Friedrich, G. (2020). Sportdidaktik. In M. Rothgangel, U. Abraham, H. Bayrhuber, V. Frederking, W. Jank \& H. J. Vollmer (Hrsg.), Lernen im Fach und über das Fach hinaus. Bestandsaufnahmen und Forschungsperspektiven aus 17 Fachdidaktiken im Vergleich (S. 391-418). Münster:Waxmann.

Oliver, R., Wehby, J., \& Reschly, D. (2011). Teacher classroom management practices: effects on disruptive or aggressive student behavior. Oslo: The Campbell Collaboration.

Oser, F. (1998). Ethos - die Vermenschlichung des Erfolgs. Zur Psychologie der Berufsmoral von Lehrpersonen. Opladen: Leske + Budrich.

Pfitzner, M., \& Schoppek, W. (2000). Gemeinsamkeiten und Diskrepanzen in der Bewertung von Unterrichtsstörungen durch Lehrer und Schüler eine empirische Untersuchung. Unterrichtswissenschaft, 18(4), 350-378.

Piwowar, V. (2013). Multidimensionale Erfassung von Kompetenzen im Klassenmanagement: Konstruktion und Validierung eines Beobachter-und eines Schülerfragebogens für die Sekundarstufe 1.Zeitschrift für pädagogische Psychologie, 27(4) 215-228.

Reuker, S. (2017a). The knowledge-based reasoning of physical education teachers: a comparison between groups with different expertise. European Physical Education Review, 23(1), 3-24.

Reuker, S. (2017b). The noticing of physical education teachers: a comparison between groups with different expertise. Physical Education and Sport Pedagogy, 22(2), 150-170.

Reuker, S. (2018). "Ich unterrichte so, wie es die Ereignisse erfordern" - Der Professionelle Blick von Sportlehrkräften und seine Bedeutung für adaptiven Unterricht. Zeitschrift für sportpädagogische Forschung, 6(2), 31-52.

Reusser, K. (2011). Von der Unterrichtsforschung zur Unterrichtsentwicklung - Probleme, Strategien, Werkzeuge. In W. Einsiedler (Hrsg.), Unterrichtsentwicklung und didaktische Entwicklungsforschung (S. 11-40). Bad Heilbrunn: Klinkhardt.

Rost, D. (2004). Lehrbuch Testtheorie - Testkonstruktion. Göttingen:Hogrefe.

Schierz, M. (2013). Bildungspolitische Reformvorgaben und fachkulturelle Reproduktionen Beobachtungen am Beispiel des Schulfaches Sport. Spectrum der Sportwissenschaften, 25(1), 64-79.

Serwe-Pandrick, E. (2013). "The reflective turn"? Fachdidaktische Positionen zu einer "reflektier- 


\section{Hauptbeitrag}

ten Praxis" im Sportunterricht. Zeitschrift für sportpädagogische Forschung, 1(2), 26-44.

Wegener, M., Wegener, M., \& Kastrup, V. (2012).

Akustische Aspekte des Sportunterrichts: leiser statt heiser. Sportunterricht, 61(9), 258-264.

Weiber, R., \& Mühlhaus, D. (2010). Strukturgleichungsmodellierung. Eine anwendungsorientierte Einführung in die Kausalanalyse mit Hilfe von AMOS, SmartPLS und SPSS. Heidelberg: Springer.

Weinert, F. E., \& Helmke, A. (Hrsg.). (1997). Entwicklung im Grundschulalter. Weinheim: Beltz.

Weinstein, C. S. (1999). Reflections of best practices and promising programs: beyond assertive classroom discipline. In H.J. Freiberg (Hrsg.), Beyond behaviorism: changing the classroom management paradigm (S. 147-163). Boston: Allyn \& Bacon.

Wiesche, D. (2013). Ich muss immer etwas vormachen, damit die anderen sehen, wie es nicht geht! Sportunterricht, 62(7), 194-199.

Wirtz, M., \& Caspar, F. (2002). Beurteilerübereinstimmung und Beurteilerreliabilität. Göttingen: Hogrefe.

Wolters, P., \& Kemna, P. (2011).Qualitätskriterienfürden Sportunterricht-Entwurfeines Rahmenmodells und einer Skala zur erlebten Sicherheit. In K. O. Bauer \& N. Logemann (Hrsg.), Unterrichtsqualität und fachdidaktische Forschung. Modelle und Instrumente zur Messung fachspezifischer Lernbedingungen und Kompetenzen (S. 159-185). Münster:Waxmann. 